\title{
MicroRNA-214-3p inhibits proliferation and cell cycle progression by targeting MELK in hepatocellular carcinoma and correlates cancer prognosis
}

Yue Li ${ }^{1}$, You Li ${ }^{2}$, Yao Chen ${ }^{1}$, Qian Xie ${ }^{1}$, Ningning Dong ${ }^{1}$, Yanjun Gao ${ }^{1}$, Huan Deng ${ }^{1}$, Chunhua Lu²* and Suihai Wang ${ }^{1 *}$ (D)

\begin{abstract}
Background: MicroRNAs are considered as potential regulators in various biological pathways and contribute to the diagnosis and prognosis of cancers. MicroRNA-214-3p (miR-214-3p) was proved to be correlated with various cancers in recent studies. However, the biological functions of miR-214-3p in hepatocellular carcinoma (HCC) and its association with the prognosis of HCC after liver transplantation are still unevaluated. Here we intended to elucidate the functional implication of miR-214-3p in regulation of cell proliferation and apoptosis and its potential prediction of clinical prognosis of HCC patients.
\end{abstract}

Methods: Expressions of miR-214-3p in 98 HCC patients and three HCC cell lines were detected by quantitative reverse transcription PCR (qRT-PCR) to explore the association of miR-214-3p expression and clinicopathological characteristics. The effects of miR-214-3p on cell proliferation and apoptosis were examined by proliferation and flow cytometry assay, respectively. The direct target gene of miR-214-3p was also detected by luciferase reporter assay.

Results: The effects of miR-214-3p on cell proliferation and apoptosis were examined by proliferation and flow cytometry assay, respectively. The direct target gene of miR-214-3p was also detected by luciferase reporter assay. The results showed that miR-214-3p expression was downregulated in primary HCC samples compared with normal liver tissues, and was decreased in HCC recurrence species compared with non-recurrence controls $(P=0.001)$. Low miR214-3p level was associated with poor overall survival $(\mathrm{OS})$ (Log rank $P=0.003$ ) and recurrence-free survival (RFS) (Log rank $P=0.007)$. Moreover, miR-214-3p precursor transfection resulted in decreased cell proliferation, cell cycle arrest at G1 phase, and enhanced cell apoptosis in HepG2 and HUH-7 cells. Further investigation showed that miR-214-3p could regulate its target gene maternal embryonic leucine zipper kinase (MELK) by directly binding to MELK-3'-UTR.

Conclusions: miR-214-3p suppresses HCC progression by directly down-regulating MELK expression, indicating a potential therapeutic target for the treatment and prognosis of HCC patients.

Keywords: microRNA-214-3p, MELK, Hepatocellular carcinoma, Recurrence, Proliferation, Apoptosis, Cell cycle arrest

\footnotetext{
*Correspondence: yuze_ma@163.com; suihaiw65@163.com

${ }^{1}$ Institute of Antibody Engineering, Department of Laboratory Medicine,

Nanfang Hospital, Southern Medical University, No. 1838, Guangzhou

Avenue North, Guangzhou 510515, China

2 Department of Biotechnology, College of Life Science and Technology,

Guangxi University, No. 100, Daxue Road, Nanning 530004, Guangxi

Province, China
} 


\section{Background}

Hepatocellular carcinoma ( $\mathrm{HCC}$ ) is the third leading cause of cancer mortality worldwide with poor survival and unsatisfied prognosis [1-3]. Over decades, the incidence of HCC has been dramatically increased especially in hepatitis B or C virus (HBV or HCV) infection induced phenotype [4]. Until now, surgical hepatic resection and liver transplantation are still the main curative treatment for HCC patients. Although great advances have been made in treatment and diagnosis, the prognosis of HCC remains limited, with its survival rates under $20 \%$ at 5 years [5]. Therefore, it's an urgent need to understand the molecular mechanisms responsible for the pathogenesis of HCC and to identify effective treatment strategies. However, tumor recurrence rates remain a major concern for the exhibition of active hepatitis or cirrhosis in surrounding non-tumor liver tissues, even in patients who have received curative treatments $[6,7]$. A better understanding of the molecular mechanisms that can distinguish progressive from non-progressive $\mathrm{HCC}$ is indispensable for exploring novel prognostic markers and therapeutic targets which may guide the surveillance after liver transplantation.

Recent evidences support that microRNAs (miRNAs) serve as potential indicators for diagnosis and prognosis of cancers [8]. miRNAs are small noncoding RNAs, which contain $20 \sim 23$ nucleotides, processed from primiRNAs and contribute to post-transcriptional regulation of target gene expression through binding directly to the specific sequences of target genes' $3^{\prime}$-UTRs [8]. Researches show that miRNAs have effects on cell proliferation, migration, and apoptosis via making a difference in the stability or translation of target mRNA. Additionally, miRNAs are considered as crucial participators in tumor progression through influencing multiple biological functions and pathways [9]. Previous study has demonstrated that miR-214-3p is correlated with tumor onset and progression [10]. It has been reported that miR-214-3p is downregulated in HCC tissues and closely related to fibrotic stages $[11,12]$; however, the biological functions of miR-214-3p in HCC and its position in $\mathrm{HCC}$ prognosis after transplantation remain unclear.

In our current study, the expression of miR-214-3p in the formalin-fixed paraffin-embedded (FFPE) tumor tissues from HCC patients was detected, and the correlation of miR-214-3p expression with lymph node metastasis, recurrence, pathological $\mathrm{T}$ stage, and age was analyzed as well. Moreover, a further insight into the function of miR-214-3p in regulating HCC cell proliferation, cell cycle, and apoptosis was gained by overexpressing miR-214-3p in human HCC cells.

\section{Methods}

Patients and tissue samples

A cohort of 98 patients undergoing liver transplantation for HCC was obtained with their follow-up data from the Nanfang Hospital of Southern Medical University from January 2006 to November 2011. All the patients were followed until December 2011. The median recurrencefree period was 12 months for patients with HCC recurrence compared to 65 months for patients without HCC recurrence. All of these 98 patients enrolled in this study met the transplantation criteria for HCC [13]. HCC samples were from the paraffin embedded archival tissue blocks and the normal liver tissues were from the liver hemangioma resection. The clinicopathological parameters of patients with HCC were summarized in Table 1. Informed consents from all patients were provided according to the protocols approved by the Institutional Review Boards of the Nanfang Hospital of Southern Medical University.

\section{Cell culture and transfection}

HepG2, HUH-7, SNU398 and L-O2 cell lines used in this study were purchased from the ATCC (Manassas, VA). All cells then were cultured in EMEM and supplemented with $10 \%(\mathrm{v} / \mathrm{v})$ heat-inactivated fetal bovine serum (FBS, Gemini Bio-Products, Sacramento, CA) and antibiotics (98 U/ml penicillin and $98 \mu \mathrm{g} / \mathrm{ml}$ streptomycin) at $37^{\circ} \mathrm{C}$ in a humidified atmosphere of $5 \% \mathrm{CO} 2$. Double-stranded RNAs that mimic endogenous precursor miR-214-3p (Invitrogen-Life Technologies, Carlsbad, CA) as well as negative oligonucleotide control was transfected into cells using Oligofectamine (Thermo Scientific, Waltham, MA) according to the manufacturer's instruction.

\section{RNA isolation and Taqman real-time PCR}

Total RNA was isolated and then reverse transcribed to cDNA with the stem-loop RT primer for miR-214-3p. miR-214-3p expression was normalized and quantificated using U6 small RNA as an internal control. For miR214-3p analysis, primers were $5^{\prime}$-GCATCCTGCCTCC ACATGCAT- $3^{\prime}$ and 5'-GCGCTGAGGAATAATAG AGTATGTAT- $3^{\prime}$. PCR primers for the internal control U6 were $5^{\prime}$-TGACTTCCAAG TACCATCGCCA- ${ }^{\prime}$ and $5^{\prime}$-TTGTAGAGGTAGGTGTGCAGCAT- ${ }^{\prime}$. The relative expression levels were calculated using the $2-\Delta \Delta \mathrm{Ct}$ method. All the experiments were run in triplicate.

\section{Cell proliferation assay}

Cell proliferation assay was carried out using cell Titer 96 Aqueous one Solution Cell Proliferation Assay (Promega, Madison, WI) follow in the manufacturer's protocol. Three independent experiments were done. 
Table 1 Clinicopathology parameters in 98 HCC patients according to high- or low miR-214-3p expression level

\begin{tabular}{|c|c|c|c|c|}
\hline Parameter & $\mathrm{n}$ & $\begin{array}{l}\text { Low miR-214-3p } \\
\text { expression }\end{array}$ & $\begin{array}{l}\text { High miR- } \\
214-3 p \\
\text { expression }\end{array}$ & $\mathbf{P}$ \\
\hline Age & 98 & $56.60 \pm 6.14$ & $54.06 \pm 8.73$ & $0.327^{*}$ \\
\hline \multicolumn{5}{|l|}{ Gender } \\
\hline Male & 83 & 44 & 39 & \multirow[t]{2}{*}{$0.742^{\#}$} \\
\hline Female & 15 & 6 & 9 & \\
\hline \multicolumn{5}{|l|}{ Liver disease } \\
\hline HBV & 93 & 46 & 47 & \multirow[t]{2}{*}{$1.000^{4}$} \\
\hline Others & 5 & 3 & 2 & \\
\hline \multicolumn{5}{|l|}{ Cirrhosis } \\
\hline Positive & 94 & 46 & 48 & \multirow[t]{2}{*}{$1.000^{4}$} \\
\hline Negative & 4 & 2 & 2 & \\
\hline \multicolumn{5}{|l|}{ Tumor stage } \\
\hline $1+\|$ & 65 & 32 & 33 & \multirow[t]{2}{*}{$0.791^{\#}$} \\
\hline III & 33 & 18 & 15 & \\
\hline \multicolumn{5}{|l|}{ Histologic grade } \\
\hline Differentiated & 87 & 41 & 46 & \multirow[t]{2}{*}{$0.068^{4}$} \\
\hline Undifferentiated & 11 & 8 & 3 & \\
\hline \multicolumn{5}{|c|}{ Milan criteria } \\
\hline $\ln$ & 53 & 23 & 30 & \multirow[t]{2}{*}{$0.163^{\#}$} \\
\hline Out & 45 & 26 & 19 & \\
\hline \multicolumn{5}{|l|}{ Tumor size (cm) } \\
\hline$\leq 4.5$ & 59 & 24 & 35 & \multirow[t]{2}{*}{$0.016^{\#}$} \\
\hline$>4.5$ & 39 & 25 & 14 & \\
\hline \multicolumn{5}{|l|}{ Multinodular } \\
\hline Positive & 43 & 25 & 18 & \multirow[t]{2}{*}{$0.044^{\#}$} \\
\hline Negative & 55 & 24 & 31 & \\
\hline \multicolumn{5}{|l|}{ Vascular invasion } \\
\hline Positive & 22 & 16 & 6 & \multirow[t]{2}{*}{$0.025^{\#}$} \\
\hline Negative & 77 & 33 & 43 & \\
\hline \multicolumn{5}{|c|}{ Serum AFP (ng/ml) } \\
\hline$\leq 400$ & 62 & 29 & 33 & \multirow[t]{4}{*}{$1.000^{\#}$} \\
\hline$>400$ & 36 & 19 & 17 & \\
\hline Overall survival & $41 / 98$ & $11 / 49$ & $30 / 49$ & \\
\hline HCC recurrence & $57 / 98$ & $37 / 49$ & $20 / 49$ & \\
\hline
\end{tabular}

AFP alpha-fetoprotein

* Unpaired student t-test; ${ }^{*}$ Chi square test; ${ }^{\circledR}$ Fisher's exact test

\section{Cell cycle analysis}

HepG2 and HUH-7 cells were collected in the log phase of growth and incubated for $24 \mathrm{~h}$. Then the cells were trypsinized, washed with PBS twice, and fixed overnight in cold $75 \%$ ethanol at $4{ }^{\circ} \mathrm{C}$. After that, the fixed cells were stained with propidium iodide, follow by examination using flow cytometer (BD Biosciences, San Jose,
CA). Finally, DNA histograms were analyzed with modified software. Three independent experiments were done.

\section{Apoptosis analysis}

A total of $5 \times 10^{5}$ cells were harvested and centrifuged for apoptotic evaluation. Propidium iodide (BD Bioscience) and the fluorescein isothiocyanate-conjugated (FITC) anti-human Annexin V Apoptosis Detection Kit I (BD Pharmingen) was used to characterize cells according to the manufacturer's instructions. Labeled cells were detected using the fluorescence activated cell sorting (FACS) Aria II Cell Sorter System (BD Biosciences), followed by data analysis using the Diva program (BD Biosciences). Three independent experiments were done.

\section{Luciferase activity assay}

For luciferase reporter assay, HEK293T cells were cultured in 48-well plates and then cotransfected with $10 \mathrm{ng}$ pGL3 cm-MELK-3' UTR-Wt or pGL3 cm-MELK-3' UTR-Mut, 30 pmol of miR-214-3p precursor or NC oligonucleotides, and 2 ng of pRL-TK (Ruibo, Guangzhou, China). After transfection for $72 \mathrm{~h}$, cells were collected separately and then analyzed following the Dual-Luciferase Reporter Assay protocol (Promega, Madison, WI). The data were presented as relative luciferase activity. Three independent experiments were done.

\section{Immunohistochemical (IHC) staining}

Briefly, before antigen retrieval in citrate buffer, tissue sections were dewaxed and subsequently rehydrated in graded series of ethanols. After that, the sections were incubated overnight with antibody against MELK (Epitomics, Burlingame, USA; $1: 200)$ at $4{ }^{\circ} \mathrm{C}$, followed by incubation with an HRP-conjugated secondary antibody and DAB (Dako, Carpenteria, CA). DAB was used for color development, compared with dark brown staining was considered positive.

\section{Western blot analysis}

Cells were harvested and lysed in lysis buffer supplemented with proteinase inhibitor cocktail on ice for $20 \mathrm{~min}$. Cell lysates were resolved by SDS-PAGE and transferred to PVDF membranes (Millipore). The membranes were blocked for $1 \mathrm{~h}$ in 5\% non-fat dry milk and incubated with primary MELK (Epitomics, Burlingame, USA or GAPDH (Cell signaling technology, Danvers, USA) antibodies at $4{ }^{\circ} \mathrm{C}$ overnight. Then the membranes were incubated with HRP-conjugated secondary antibodies and detected with ECL Plus (Millipore). 


\section{Statistical analysis}

The SPSS version 17.0 (SPSS Inc. Chicago, IL) was used for statistical analysis in this study. Comparisons between two groups were performed using Student's $t$ test. Correlations between clinicopathologic characteristics and immunohistochemical variables were analyzed using Chi square test or Fisher's exact test. The Kaplan-Meier method was selected to graph survival curves. Log-rank statistic was applied to calculate the differences between the groups. The impact of prognostic factors on RFS and OS were analyzed by Cox proportional hazard models. A two-sided $P$ value less than 0.05 was considered statistically significant.

\section{Results}

\section{Downregulation of miR-214-3p in primary HCC tissues}

We first determined miR-214-3p expression in HCC cell lines (HepG2, HUH-7 and SNU398) and a normal hepatic cell line (L-O2). Our results showed that miR214-3p exhibited decreased expressions in HCC cell lines compared with L-O2 cells (Fig. 1a). To explore the clinical significance of miR-214-3p in HCC, the expression of miR214-3p was examined in HCC tissues. The results showed that miR-214-3p expression in HCC tissues $(\mathrm{n}=98)$ was dramatically lower in tumor recurrence patients than in non-recurrence patients $(\mathrm{P}=0.020$; Fig. $1 \mathrm{~b})$. Similarly, a significant reduction of miR-214-3p expression was observed in liver transplant recipients who died compared with that in the survived patients $(\mathrm{P}=0.003$; Fig. 1c).

\section{Association of miR-214-3p expression} with clinicopathological variables

In this study, we found that the decreased expression of miR-214-3p in HCC was closely link to various pathologic parameters, including tumor size $(\mathrm{P}=0.016)$, multinodular HCC $(\mathrm{P}=0.044)$, and vascular invasion $(\mathrm{P}=0.025$, Table 1$)$.

\section{Correlation of miR-214-3p in HCC with tumor recurrence and poor prognosis}

Kaplan-Meier analysis showed that decreased miR214-3p expression was connected with shorter OS (median survival 27 vs. 63 months, $\mathrm{P}=0.003$ ) and RFS (median survival 32 vs. 53 months $\mathrm{P}=0.007$ ) of liver transplant recipients (Fig. $2 \mathrm{a}$ and b). In multivariate analysis using a continuous variable Cox proportional hazards model, miR-214-3p expression was found to be predictive of outcomes independent of gender, age, and tumor stages $(\mathrm{OS}, \mathrm{P}=0.004$; RFS, $\mathrm{P}=0.018$ ).

\section{Effects of Pre214-3p transfection on cell proliferation}

To uncover whether overexpression of miR-214-3p had an effect on cell proliferation, HepG2 and HUH-7 cells were transfected with miR-214-3p precursor (Pre2143p) (Fig. 3a and b). Cell proliferation assay showed that the proliferation rate was decreased in HepG2 and HUH-7 cells with the inhibitory efficiencies were 42.5 and $36.2 \%$ after Pre214-3p transfection, respectively (Fig. 3c). Cell cycle analysis showed that compared with the control group, the percentages of G1-phase cells were increased in Pre214-3p-transfected HepG2 $(\mathrm{P}=0.005)$ and HUH-7 cells $(\mathrm{P}=0.012)$, while the percentages of $\mathrm{S}$-phase cells were decreased both cell lines $(P=0.007$ and $P=0.018)$ (Fig. $3 \mathrm{~d}$ and e). Nevertheless, no statistical significance was found in the percentages of G2/M phase cells in HepG2 or HUH-7 cells compared with the control group (Fig. $3 \mathrm{~d}$ and e). These
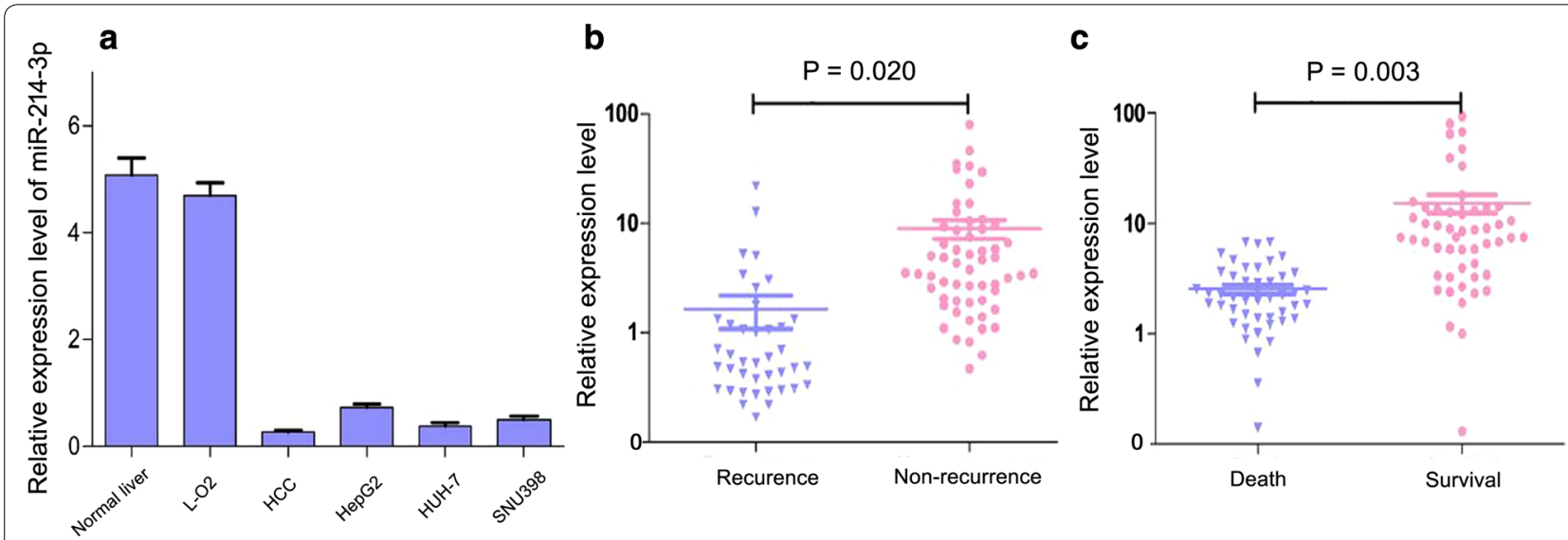

Fig. 1 MiR-214-3p expression in HCC tissues and cell lines. a Expression of miR-214-3p in 98 HCC species, normal liver tissues, LO2, HUH-7, and SNU398 cell lines detected by qRT-PCR; b Expression of miR-214-3p in HCC samples of patients with or without tumor recurrence; $\mathbf{c}$ Expression of miR-214-3p in HCC samples of patients dead and survival 

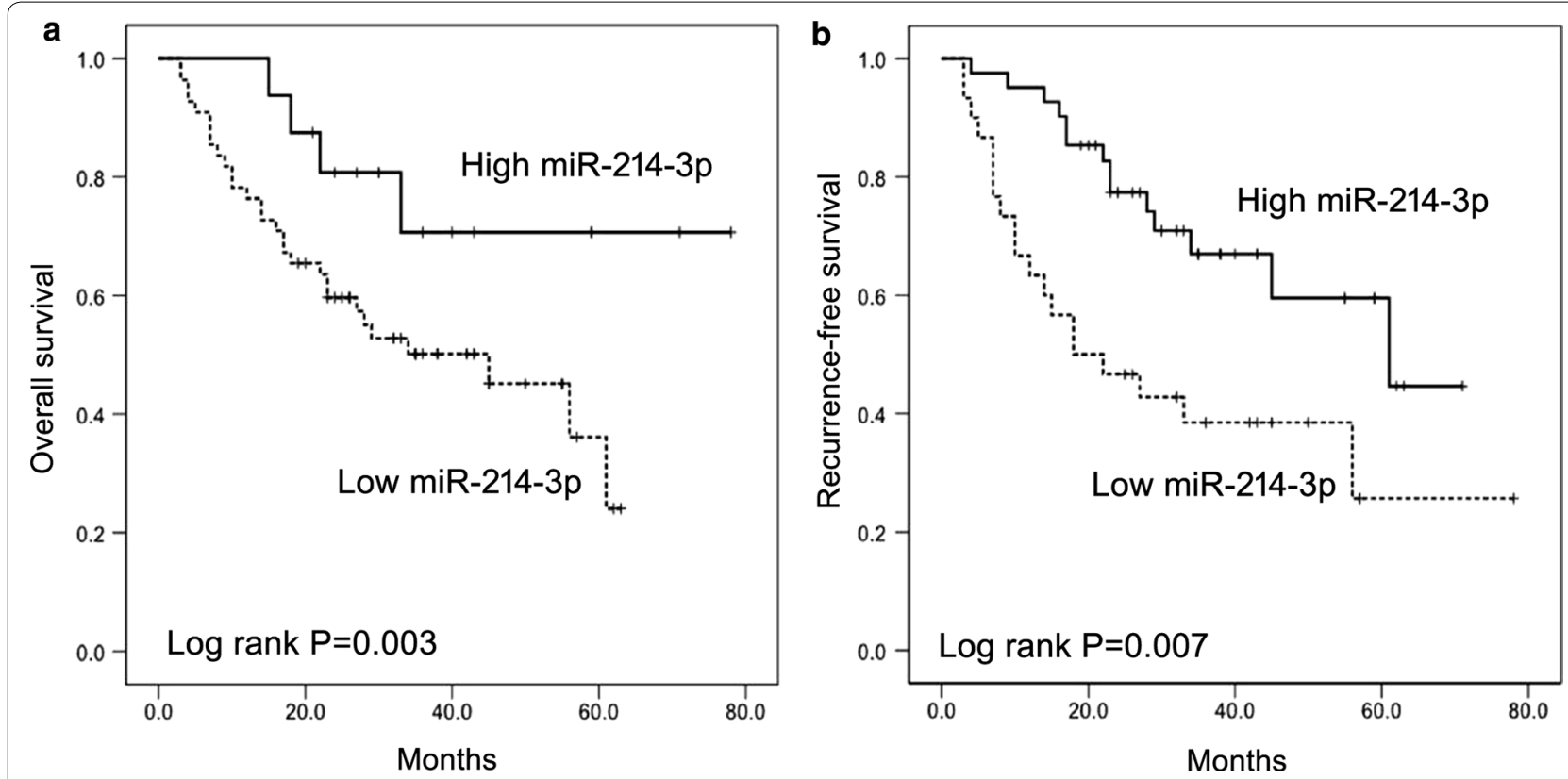

Fig. 2 Kaplan-Meier analyses of OS and RFS in 98 patients with HCC in high and low expression levels of miR-214-3p. a OS; b RFS. HCC patients were divided into miR-214-3p high and low expression groups based on the median fold-change values

results suggested that overexpression of miR-214-3p blocks cell cycle progression by inducing cell cycle arrest at $\mathrm{G} 1$ phase.

\section{Effects of Pre214-3p transfection on apoptosis}

Flow cytometry analysis was conducted to investigate whether miR-214-3p could induce apoptosis of HCC cells. Increasing apoptotic rates were observed in Pre2143p-transfected HUH-7 (P = 0.012) and HepG cells $(\mathrm{P}=0.008)$, compared with the control group (Fig. $4 \mathrm{a}$ and $b$ ).

\section{MiR-214-3p downregulates MELK expression by directly targeting its $3^{\prime}$-UTR}

Moreover, potential targeted genes of miR-214-3p were predicted using online databases, i.e. TargetScan, PicTar, and miRanda. MELK was chosen for further experimental validation due to its frequent overexpression detected by the three databases and its unknown anti-apoptotic function. Dual-luciferase reporter analysis showed that enforced expression of miR-214-3p remarkably reduced the luciferase activity of the reporter gene with the WT construct rather than with the mutant MELK 3'-UTR construct (Fig. 5a and b). Moreover, overexpression of miR-214-3p in HepG2 and HUH-7 resulted in a reduced MELK expression (Fig. 5c). Similarly, HCC sample with decreased miR-214-3p showed higher MELK expression (Fig. 5d). Together, these results suggest that miR-214-3p downregulates MELK expression by targeting MELK 3'-UTR.

\section{Discussion}

In recent decades, miRNAs have been considered as contributive regulators involved in transcriptional regulation, cell differentiation, tumorigenesis, and other biological processes [14]. Globally aberrant miRNA expression profiles of tumors have provided valuable insights into the molecular pathways of oncogenesis [15]. Nowadays, more than 2000 human miRNAs have been reported as regulational factors in cell proliferation, migration, and invasion of tumors [16]. Newly papers indicated that upregulation of miR-214-3p was found 


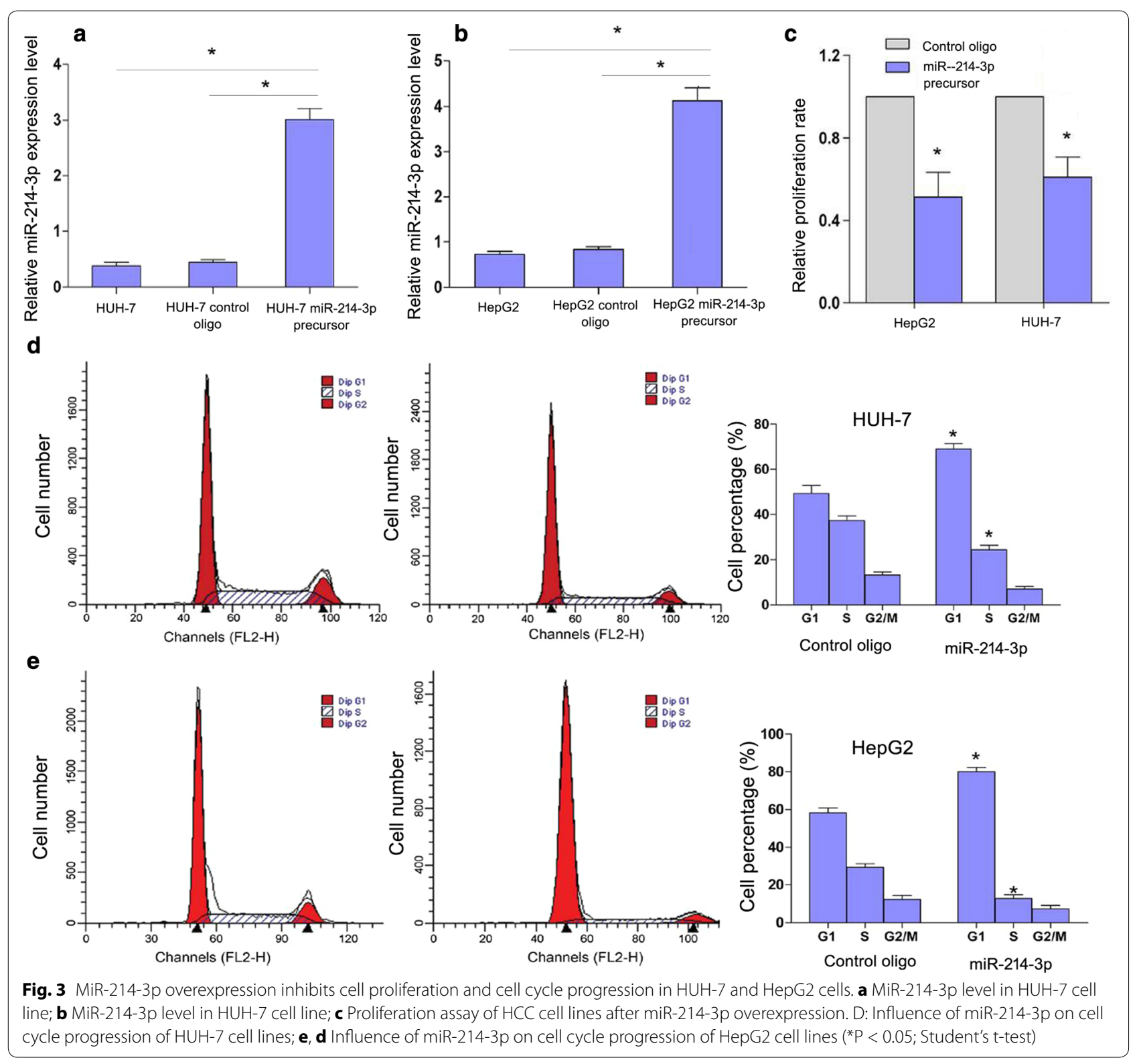

in breast cancer patients with osteolytic bone metastasis, and a knock-in miR-214-3p remarkably increased bone resorption by straightly targeting Traf3 to promote osteoclast activity and bone-resorbing activity $[17,18]$. However, other studies demonstrated that miR-214-3p was significantly downregulated in two esophageal squamous cancer cell lines compared with esophageal epithelial cells [19]. Further investigations pointed out that downregulation of miR-214-3p suppressed chemoresistance in esophageal cancer cells by targeting both survivin and CUG-BP1 [20]. The anti-apoptotic Bcl-2 family member MELK/A1 is one of these PRDI-BF1/Blimp-1 target genes. MELK as a target of miR-214-3p provides a novel perspective on the mechanisms underlying HCC proliferation and resistance to apoptosis [21]. Abundant MELK expression was detected in the bone marrow 


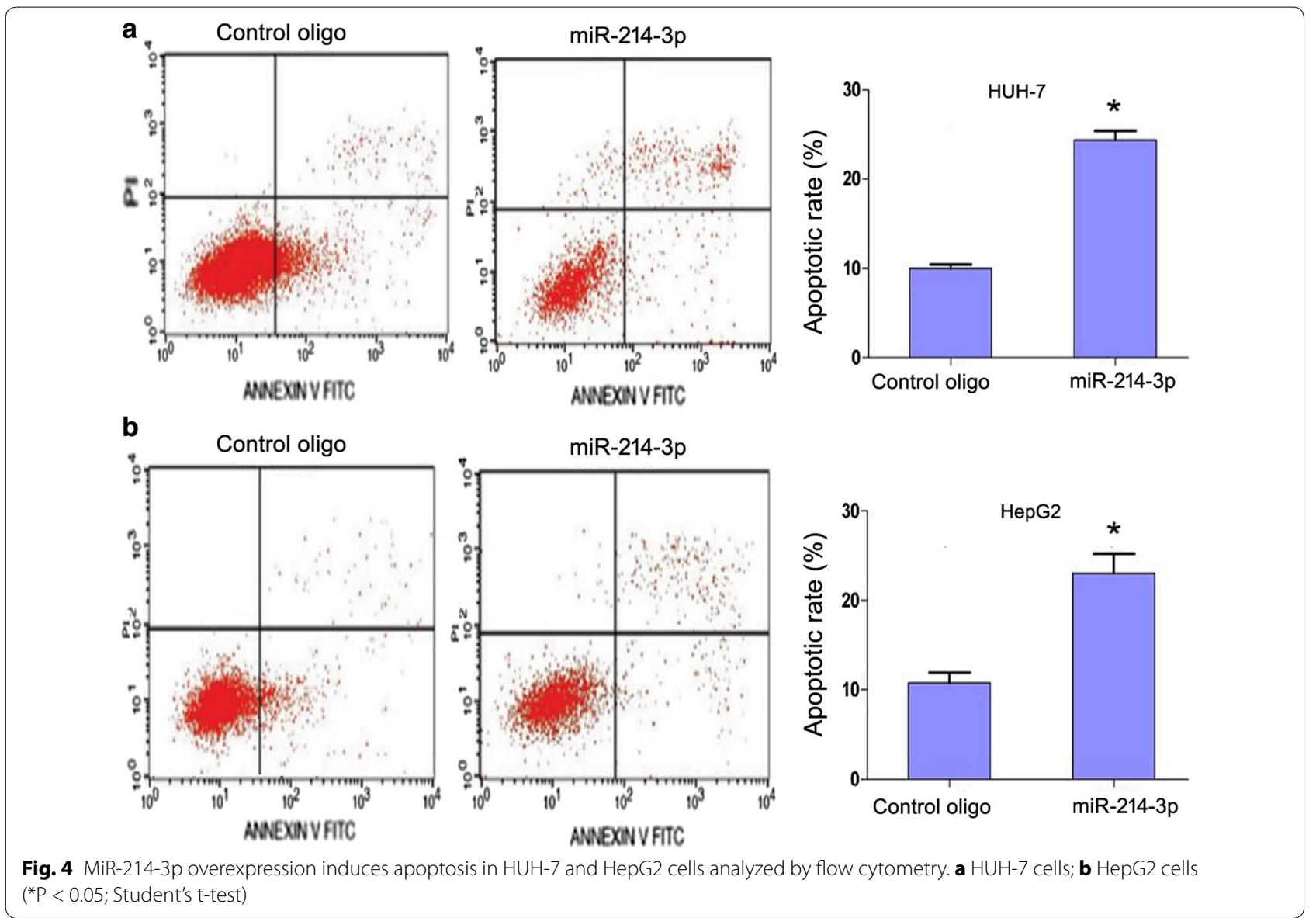

as well as in some other tissues [22, 23]. Specifically, a connection was found in clinical samples between the MELK expression and the progression of stomach carcinoma [24].

Our studies showed that miR-214-3p expression was decreased in both HCC tissues and HCC cell lines, which were consistent with previous results [11]. It has been reported that miR-214-3p expression is strongly related to with fibrotic stages [12]. Interestingly, in this study, we found that miR-214-3p expression is also closely associated with recurrence and living status of liver transplant patients. Moreover, downregulation of miR-214-3p was associated with poor survival and tumor recurrence in HCC patients. Moreover, miR-214-3p restoration inhibited cell cycle progression and accelerated apoptosis in vitro. To our best knowledge, this is the first study showing that miR-214-3p regulates cellular proliferation in HCC cells and links to the prognosis of HCC. In all, this study shown that miR-214-3p was decreased in HCC tissues and the expression level of miR-214-3p might be a significant prognostic marker for HCC patients. Based on gain-of-function approach, it is suggested that miR-214-3p could remarkably block HCC cell proliferation and induce apoptosis in vitro by directly targeting MELK $3^{\prime}$-UTR. 


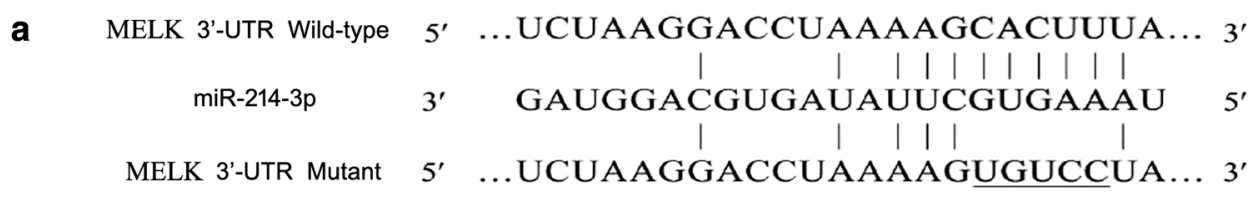

b
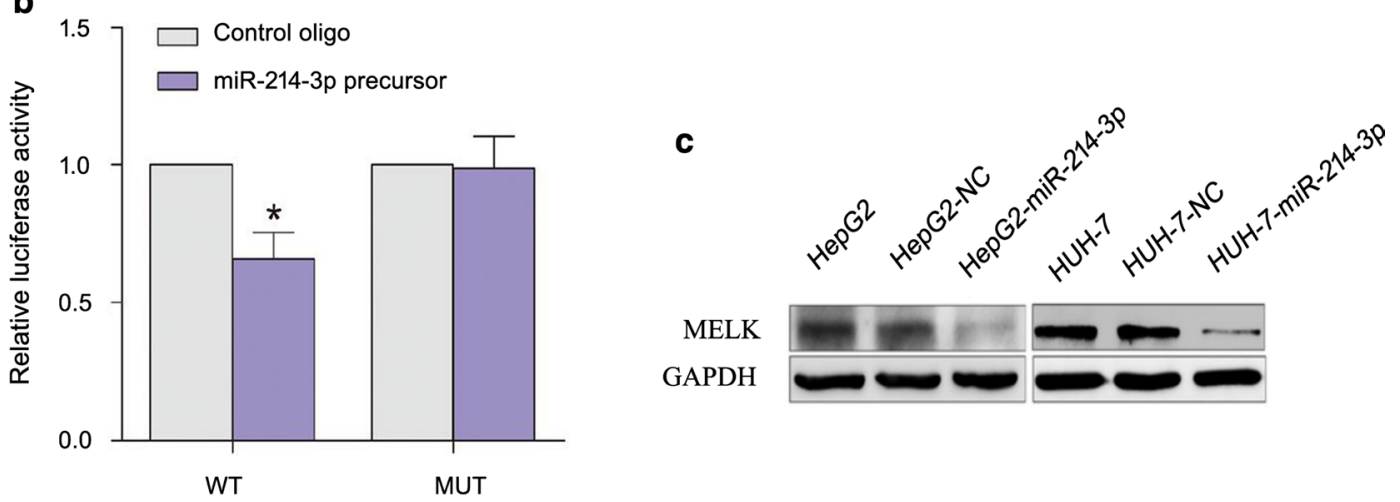

d
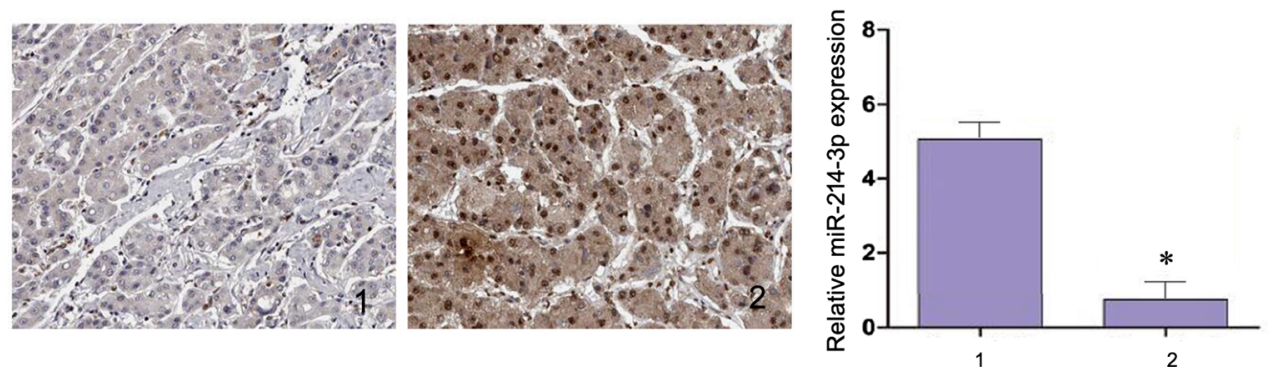

Fig. 5 MiR-214-3p down-regulates MELK expression. a WT and MUT of putative miR-214-3p target sequences of MELK $3^{\prime}-U T R ;$ b WT and MUT miR-214-3p target sequences were cotransfected with miR-214-3p precursor into HEK293T cells.; c Effects of miR-214-3p overexpression on the expression level of MELK in HepG2 and HUH-7 cells transfected with miR-214-3p by Western blot; d Expression of MELK and miR-214-3p in HCC tissues detected. Protein expression of MELK in HCC samples 1 and 2 was detected by IHC $(\times 200)$ while expression of miR-214-3p was determined using real-time PCR. 1, 2 refer to HCC sample 1 and 2. ( ${ }^{*} P<0.05$; Student's t-test)

\section{Conclusions}

Collectively, our data not only demonstrates novel insights regarding miR-214-3p function and the potential mechanisms of HCC cell proliferation, but also indicates a possible regulation pathway for MELK and a potential therapeutic strategy for HCC treatment.

\section{Abbreviations}

HCC: hepatocellular carcinoma; miR-214-3p: microRNA-214-3p; qRT-PCR: quantitative reverse transcription PCR; OS: overall survival; RFS: recurrence-free survival; MELK: maternal embryonic leucine zipper kinase; FITC: fluorescein isothiocyanate-conjugated; FACS: fluorescence activated cell sorting.

\section{Authors' contributions}

LY carried out the experiments, and also revised the manuscript. LY and CY carried out the experiments and drafted the manuscript. XQ, DNN, DH and GYJ carried out the experiments. LCH participated in the design of the study and performed the statistical analysis. WSH conceived of the study, and participated in its design and coordination and helped to draft the manuscript. All authors read and approved the final manuscript.

\section{Acknowledgements}

The authors thank statistical assistance provided by the Department of Laboratory Medicine, The Second Hospital of Hebei Medical University.

\section{Competing interests}

The authors declare that they have no competing interests.

Availability of data and materials

Please contact author for data requests.

\section{Consent for publication}

Informed consent was obtained from all individual participants included in the study.

\section{Ethics approval and consent to participate}

Ethical approval for this study was obtained from the institutional review board of the Nanfang Hospital of Southern Medical University.

\section{Funding}

This study was supported by the National Natural Science Foundation of China (No. 81201663) and the National Natural Science Foundation of China (No. 81360324). 


\section{Publisher's Note}

Springer Nature remains neutral with regard to jurisdictional claims in published maps and institutional affiliations.

Received: 28 June 2017 Accepted: 26 October 2017

Published online: 07 November 2017

\section{References}

1. Song Y, Wang F, Huang Q, et al. MicroRNAs contribute to hepatocellular carcinoma. Mini Rev Med Chem. 2015;15(6):459-66.

2. Giannelli G, Villa E, Lahn M. Transforming growth factor- $\beta$ as a therapeutic target in hepatocellular carcinoma. Cancer Res. 2014;74(7):1890-4.

3. Can A, Dogan E, Bayoglu IV, et al. Hepatocellular carcinoma in children. Asian Pac J Cancer Prev. 2014;15(6):2923-7.

4. Kakar S, Grenert JP, Paradis V, et al. Hepatocellular carcinoma arising in adenoma: similar immunohistochemical and cytogenetic features in adenoma and hepatocellular carcinoma portions of the tumor. Mod Pathol. 2014;27(11):1499-509.

5. Venook AP, Papandreou C, Furuse J, de Guevara LL. The incidence and epidemiology of hepatocellular carcinoma: a global and regional perspective. Oncologist. 2010;15(Suppl 4):5-13.

6. Couto CA, Gelape CL, Calmet F, Martin P, Levy C. Effect of ethnicity on liver transplant for hepatocellular carcinoma. Exp Clin Transplant. 2013;11(4):339-45.

7. Osório FM, Lauar GM, Lima AS, et al. Epidemiological aspects of hepatocellular carcinoma in a referral center of Minas Gerais, Brazil. Ara Gastroenterol. 2013;50(2):97-100.

8. Liu L, Chen L, Wu X, et al. Low-doseDNA-demethylating agent enhances the chemosensitivity of cancer cells by targeting cancer stem cells via the upregulation of microRNA-497. J Cancer Res Clin Oncol. 2016;142(7):1431-9.

9. Hammond SM. An overview of microRNAs. Adv Drug Deliv Rev. 2015;29(87):3-14

10. Bourassa MW, Ratan RR. MicroRNA therapeutics in neurological disease Neurochem Int. 2014;77:33-9.

11. Shi KQ, Lin Z, Chen XJ, Song M, Wang YQ, Cai YJ, Yang NB, Zheng MH, Dong JZ, Zhang L, Chen YP. Hepatocellular carcinoma associated microRNA expression signature: integrated bioinformatics analysis, experimental validation and clinical significance. Oncotarget. 2015;6(28):25093-108.
12. Chen R, Wu JC, LiuT, QuY, Lu LG, Xu MY. MicroRNA profile analysis in the liver fibrotic tissues of chronic hepatitis B patients. J Dig Dis. 2017;18(2):115-24

13. Iorio MV, Ferracin M, Liu CG, et al. MicroRNA gene expression deregulation in human breast cancer. Cancer Res. 2005;65(16):7065-70.

14. Kinose Y, Sawada K, Nakamura K, Kimura T. MicroRNA signatures in human cancers. Biomed Res Int. 2014;2014:249393.

15. Bloomston M, Frankel WL, et al. MicroRNA expression patterns to differentiate pancreatic adenocarcinoma from normal pancreas and chronipancreatitis. JAMA. 2007;297(17):1901-8.

16. Wu J, Shen $L$, Chen J, Xu H, Mao L. The role of microRNAs in enteroviral infections. Braz J Infect Dis. 2015;19(5):510-6.

17. Liu J, Li D, Dang L, et al. Osteoclastic miR-214 targets TRAF3 to contribute to osteolytic bone metastasis of breast cancer. Sci Rep. 2017;10(7):40487.

18. Gao K, Yin J, Dong J. Deregulated WWOX is involved in a negative feedback loop with microRNA-214-3p in osteosarcoma. Int J Mol Med. 2016;38(6):1850-6.

19. Irie K, Tsujimura K, Nakashima H, Nakashima K. MicroRNA-214 promotes dendritic development by targeting the schizophrenia-associated gene quaking (Qki). J Biol Chem. 2016;291(26):13891-904.

20. Phatak P, Byrnes KA, Mansour D, Liu L, Cao S, Li R, et al. Overexpression of miR-214-3p in esophageal squamous cancer cells enhances sensitivity to cisplatin by targeting survivin directly and indirectly through CUG-BP1. Oncogene. 2016;35(16):2087-97.

21. Comerford SA, Clouthier DE, Hinnant EA, Hammer RE. Induction of hepatocyte proliferation and death by modulation of T-Antigen expression. Oncogene. 2003;22(16):2515-30.

22. Xia H, Kong SN, Chen J, Shi M, Sekar K, Seshachalam VP, et al. MELK is an oncogenic kinase essential for early hepatocellular carcinoma recurrence. Cancer Lett. 2016;383(1):85-93.

23. Wright LM, Maloney W, Yu X, et al. Stromal cell-derived factor-1 binding to its chemokine receptor CXCR4 on precursor cells promotes the chemotactic recruitment, development and survival of human osteoclasts. Bone. 2005;36(5):840-53.

24. Kato T, Inoue H, Imoto S, Tamada Y, Miyamoto T, et al. Oncogenic roles of TOPK and MELK, and effective growth suppression by small molecular inhibitors in kidney cancer cells. Oncotarget. 2016;7(14):17652-64.

\section{Submit your next manuscript to BioMed Central and we will help you at every step:}

- We accept pre-submission inquiries

- Our selector tool helps you to find the most relevant journal

- We provide round the clock customer support

- Convenient online submission

- Thorough peer review

- Inclusion in PubMed and all major indexing services

- Maximum visibility for your research

Submit your manuscript at www.biomedcentral.com/submit
BioMed Central 